\title{
Paradoxical Embolism Causing Myocardial Infarction
}

\author{
Robert T. Nguyen a, Carlos Morales-Mangual ${ }^{\mathrm{a}, \mathrm{c}}$, Steven Gu${ }^{\mathrm{a}}$, Vaibhavi Uppin ${ }^{\mathrm{a}}$, \\ Joshua Jackson a, Oluwaseun Oluwo ${ }^{a}$, Snow Trinh T. Nguyen ${ }^{\mathrm{a}}$, \\ Ricardo Castillo ${ }^{\mathrm{a}}$, Abdullah Khan ${ }^{\mathrm{a}}$, Mohammad Zaman ${ }^{\mathrm{b}}$
}

\begin{abstract}
In this case report, we present a case of a likely paradoxical embolism in a young patient with no significant risk factor for coronary artery disease that initially presented with shortness of breath and bilateral calf pain that was found to have a deep vein thrombosis. There was a development of a pulmonary embolism that we believed due to right ventricular strain resulted in an embolus crossing into the arterial system via an intracardiac shunt causing a myocardial infarction via a distal occlusion of the right coronary artery. Patient was treated with full dose anticoagulation and a patent foramen ovale (PFO) closure was recommended.
\end{abstract}

Keywords: Paradoxical embolism; Myocardial infarction; Pulmonary embolism; Intracardiac shunt

\section{Introduction}

Paradoxical embolisms are embolisms that originate in the systemic venous circulation, and enter the systemic arterial circulation through a connection via an intracardiac shunt [1]. In this case report, we present a rare case of a likely paradoxical embolism in a young patient who initially presented with deep vein thrombosis, then development of pulmonary embolism (PE) causing a myocardial infarction via a distal occlusion of the right coronary artery (RCA). This is a rare case given the fact that this patient had no significant risk factor for coronary artery disease and no history or family history of thrombophilia only that was recently started on oral contraceptive pills.

\section{Case Report}

A 27-year-old female with past medical history of morbid obe-

Manuscript submitted October 8, 2017, accepted October 16, 2017

aDivision of Cardiovascular Medicine, Brookdale University Hospital, 1 Brookdale Plaza, Brooklyn, NY 11212, USA

bivision of Pulmonary and Critical Care, Brookdale University Hospital, 1 Brookdale Plaza, Brooklyn, NY 11212, USA

${ }^{\mathrm{c} C}$ Corresponding Author: Carlos Morales-Mangual, Brookdale University Hospital and Medical Center, Brooklyn, NY 11219, USA.

Email: cmorales@bhmcny.org

doi: https://doi.org/10.14740/jmc2933w sity (body mass index (BMI) of 65) and polycystic ovarian syndrome (diagnosed in 2013 and recently started on oral contraceptive pills treatment for 1 week) presented with persistent bilateral calf pain and shortness of breath for 1 day. As per patient, she had a hard time walking when going to the bathroom in the middle of the night. The bilateral calf pain was persistent on the following morning, and while on her way to the bus stop, she suddenly felt dizzy and became short of breath.

In the emergency room, her vitals showed blood pressure of $143 / 75 \mathrm{~mm} \mathrm{Hg}$, pulse of $95 \mathrm{bpm}$, respirations of 18 per minute, and temperature of $36.7^{\circ} \mathrm{C}$. Initial blood gas on $40 \% \mathrm{FiO}_{2}$ was $\mathrm{pH} 7.34, \mathrm{pCO}_{2} 36.1$, and $\mathrm{paO}_{2} 270.1$. Due to the sudden onset of chest pain, she experienced in the emergency room, serial troponins and serial electrocardiograms (EKG) were obtained. The initial troponin measured at $0.044 \mathrm{ng} / \mathrm{mL}$ with an EKG (Fig. 1) showed T-wave inversions in leads III, AVF and V2. D-dimer was 7,911. Chest radiograph showed no active disease. On physical exam, she was diaphoretic and exhibited tenderness on the lower left leg.

A CT angiogram of the chest was done which showed filling defects bilaterally. The findings were more pronounced within the right lung with involvement of segmental and subsegmental branches. Her presentation and findings were consistent for sub-massive PE. The patient was managed with a therapeutic dose of anticoagulation and was admitted to general medicine.

During her hospital course, the troponin-I peaked at 48 $\mathrm{ng} / \mathrm{mL}$. Cardiology consultation was obtained and serial EKGs were done which showed Q-waves in the inferior leads. Patient was then treated for a possible concomitant non-ST elevation myocardial infarction (NSTEMI). On transthoracic echocardiogram, the pulmonary artery pressure was $50-55 \mathrm{~mm} \mathrm{Hg}$ with a dilated right atrium and right ventricle, and reduced right ventricle systolic function. Left ventricle systolic function was normal with no wall motion abnormalities.

At that time, the differential diagnosis for the patient's elevated troponin was thought to be attributed to the right heart strain secondary to acute PE vs. acute myocardial infarction due to the significant elevation in the cardiac enzymes not usually seen with a sub-massive PE.

Due to her worsening chest pain, cardiothoracic surgery was consulted for thrombectomy but she was deemed not an optimal candidate due to the location of the PE. Interventional cardiology was consulted and recommended right and left heart catheterization.

After risks and benefits were explained, the patient refused left heart catheterization and opted only to have right heart catheterization at the time of the procedure. 


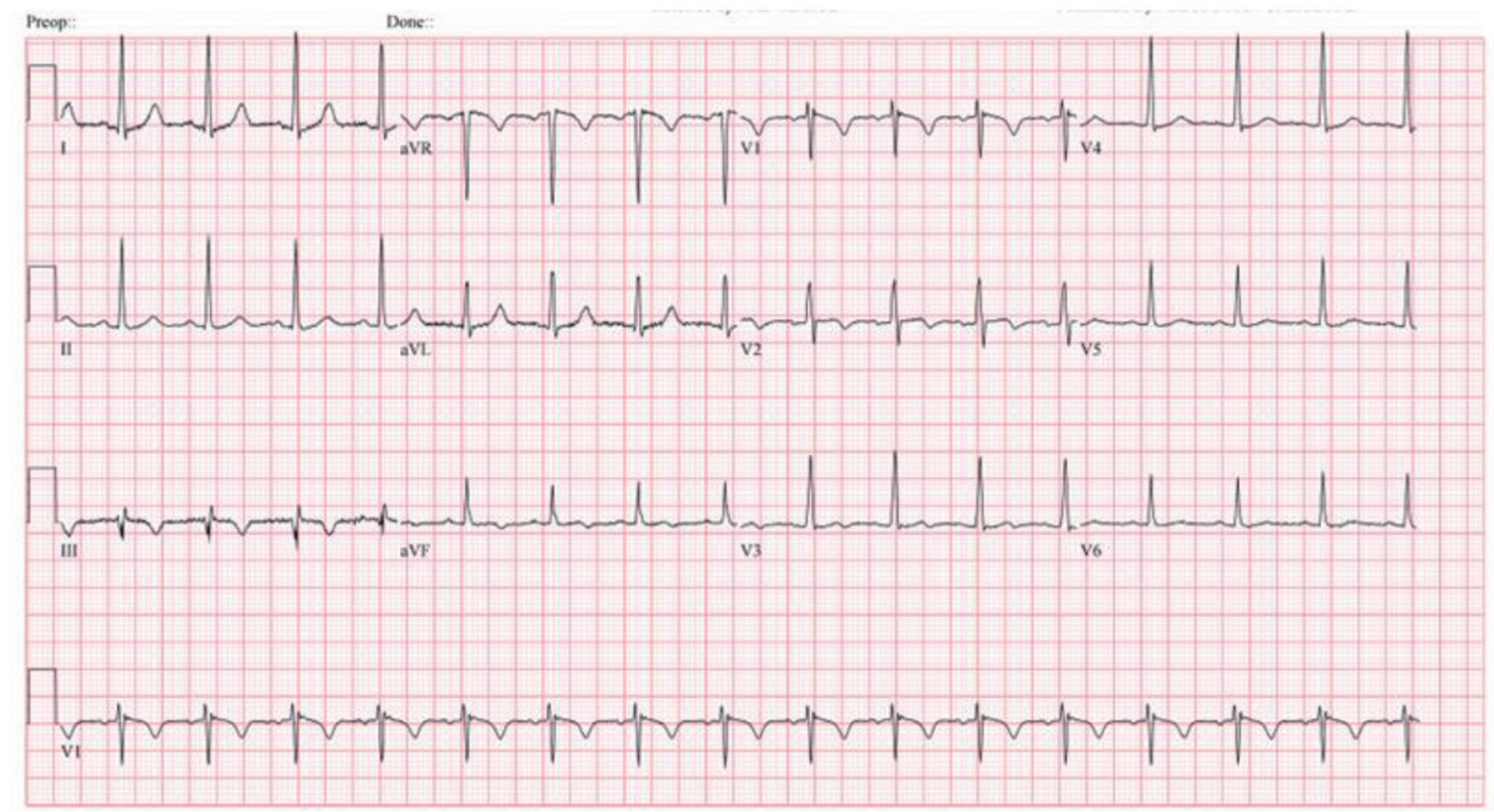

Figure 1. Electrocardiogram showing T-wave inversions in leads III, AVF and V2.

Patient then underwent catheter directed low dose thrombolytic therapy of the right pulmonary artery as well as main pulmonary trunk to complete re-canalization process.

On hospital day 4, she consented to left-sided coronary angiography, which showed a thrombus (Fig. 2) at the distal RCA extending into the posterior descending artery (PDA). With a confirmed thrombus, paradoxical embolus became the top differential diagnosis.

A subsequent echocardiogram was done to evaluate for intracardiac shunting. A significant amount of shunting (Fig. 3) was confirmed by contrast study from the right to the left atrium, suggestive of the presence of an atrial septal defect (ASD) or a patent foramen ovale (PFO). Patient was discharged 2 days later with strict instructions to continue long-term anticoagulation therapy.

\section{Discussion}

$\mathrm{PE}$ is often misdiagnosed due to multiple variable presentations. Dyspnea, tachypnea and chest pain are presenting symptoms in more than $90 \%$ of the cases. The risk factors for PE in this patient include morbid obesity, and oral contraceptive pills usage as her hypercoagulable workup was negative. The patient had no cardiovascular risk factors for myocardial infarction. CT angiogram with intravenous contrast is the gold standard to diagnose PE [2].

In general, increased troponin I levels in patients with PE can be contributed to the acute rise in pulmonary artery pressures. This leads to subsequent dilatation of the right ventricle, which causes regional right ventricular ischemia and injury without any obstructive coronary artery disease. Troponin I is a biomarker with good performance to identify right ventricular dysfunction in PE [3]. A less common cause for the elevated troponins is paradoxical embolisms, which travel from the right atrium to left atrium through a PFO or ASD. Most likely, this happened to our patient who had a new thromboembolic event after PE.

Our clinical hypothesis is that due to right ventricular

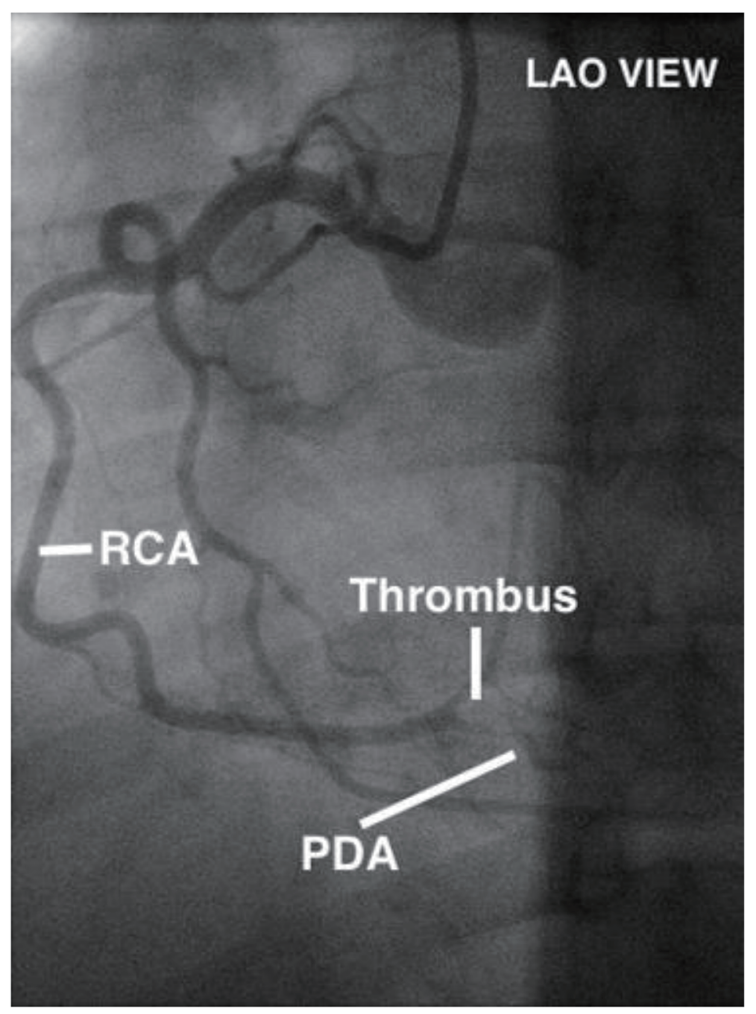

Figure 2. Patient angiogram showing thrombus in distal RCA extending into the PDA. 


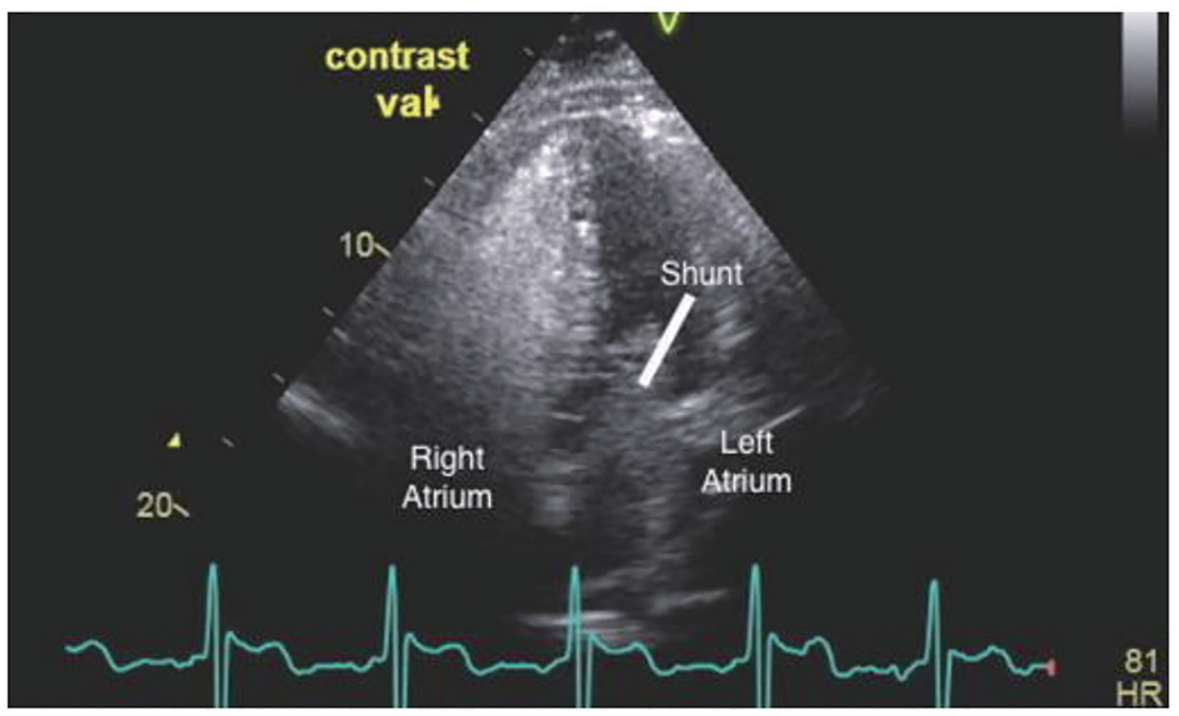

Figure 3. Transthoracic echocardiogram showing intracardiac shunt.

strain, there was increased right-sided pressure, and the change in pressure gradient in the right into the left atrium caused the sudden reversal of blood flow direction, which allowed the emboli to travel into the left circulation. Eventually, the embolus lodged into distal RCA causing myocardial infarction.

Treatment for life-threatening PE is thrombolysis with anticoagulation therapy. Cardiothoracic surgery is indicated in high-risk patients, or in patients with contraindications to thrombolytic drugs [4]. In the current literature, there is no difference in outcomes between localized intrapulmonary thrombolysis treatments compared to systemic intravenous thrombolysis treatment [5].

An updated analysis of the RESPECT trial that was presented on November 2016 showed that PFO closure with the Amplatzer PFO occluder was superior to traditional medical management in reducing recurrent ischemic strokes on longterm follow-up in patients with true cryptogenic stroke and evidence of a PFO [6]. Due to the similar etiology of the occluding embolus in the patient's right coronary, she was referred for PFO closure.

\section{References}

1. Windecker S, Stortecky S, Meier B. Paradoxical embo- lism. J Am Coll Cardiol. 2014;64(4):403-415.

2. Torbicki A, Perrier A, Konstantinides S, Agnelli G, Galie $\mathrm{N}$, Pruszczyk P, Bengel F, et al. Guidelines on the diagnosis and management of acute pulmonary embolism: the task force for the diagnosis and management of acute pulmonary embolism of the European Society of Cardiology (ESC). Eur Heart J. 2008;29(18):2276-2315.

3. Daquarti G, March Vecchio N, Mitrione CS, Furmento J, Ametrano MC, Dominguez Pace MP, Costabel JP. High-sensitivity troponin and right ventricular function in acute pulmonary embolism. Am J Emerg Med. 2016;34(8):1579-1582.

4. Uflacker R. Interventional therapy for pulmonary embolism. J Vasc Interv Radiol. 2001;12(2):147-164.

5. Verstraete M, Miller GA, Bounameaux H, Charbonnier B, Colle JP, Lecorf G, Marbet GA, et al. Intravenous and intrapulmonary recombinant tissue-type plasminogen activator in the treatment of acute massive pulmonary embolism. Circulation. 1988;77(2):353-360.

6. Kent DM, Dahabreh IJ, Ruthazer R, Furlan AJ, Reisman M, Carroll JD, Saver JL, et al. Device closure of patent foramen ovale after stroke: pooled analysis of completed randomized trials. J Am Coll Cardiol. 2016;67(8):907917. 